\title{
DYNAMIC ANALYSIS OF RAILWAY VEHICLE-TRACK INTERACTIONS DUE TO WHEEL RATWTH A PICH-PLANE VEHICLE MODEL
}

\author{
Rajib Ul Alam Uzzal, Waiz Ahmed and Subhash Rakheja \\ Department of Mechanical and Industrial Engineering \\ Concordia University, Montreal, Canada
}

\begin{abstract}
This paper presents the responses of the railway vehicle and track components in terms of contact forces and displacements. The considered vehicle model is a five-DOF pitch-plane lumped parameter quarter car model supported on two-dimensional track systems comprising three layers. The car body is linked with the vehicle bogie through secondary suspension springs and damper elements, which is further linked to the wheels through primary suspension springs and damper elements. In modeling of the track, the rail is considered as an infinitely long beam discretely supported by a series of springs, dampers and masses representing the elasticity and damping effects of the rail pads, ballasts, and subgrades respectively. The non-linear Hertzian contact theory is employed to accomplish the dynamic interactions between the lumped mass vehicle and the continuous rail. The drastic effect of one wheel flat to the other perfect wheel-rail contact point is also taken into account.
\end{abstract}

Keywords: Wheel flat, pitch-plane vehicle, wheel-rail impact, component force.

\section{INTRODUCTION}

The existence of defects in a railway wheel is one of the main sources of railway vehicle-track abnormal vibrations. Wheel flat is the most common type of wheel defect encountered by the railway industry. With the significant increase of train speed and axle load, the vibration of the coupled vehicle and track system due to wheel flat is further intensified and the safe operation of trains is reduced. Furthermore, the presence of flat in the wheel within these criteria may cause harm not only on the wheel and rail but also to the entire vehicle and track system. The repeated dynamic loads due to wheel flats will cause failure and fatigue damage of the vehicle and track components. A comprehensive study in the presence of wheel flat is thus necessary for prediction of the impact force generated at the interfaces of different components of vehicle-track system.

The dynamic interaction between the vehicle, the wheel, and the rail track systems has been the subject of extensive research in recent years. Many studies ${ }^{1,2,3}$ have focused on the vibrations of a railway track under moving vehicles with wheel defects, and different theories and models have been presented on this issue. A detailed review in the area of railway vehicle dynamics featuring the historical survey of the dynamic models to study the vehicles-track interactions due to wheel defects at high frequency range have been presented by Knothe and Grassie $^{4}$. In analysis of the coupled vehicle-track system, some of these studies ${ }^{3,5}$ have employed finite element method and some ${ }^{6,7,8}$ have used modal analysis method. Two types of track models are generally employed in the study of vehicle-track interactions. Early track system studies considering rail as a discretely supported beam ${ }^{1,9}$ is now widely used for modelling of wheel-rail interactions $2,6,7,8,10$. Vehicle-track interaction studies in general consider track as a continuous system as Euler-Bernoulli ${ }^{6,7}$, ${ }^{8}$ or Timoshenko beam ${ }^{1,2,9,10}$. Most of these studies, however, represent vehicle by a rolling wheel to investigate the wheel-rail contact forces. The impact force response such as the bearing force, rail pad force, and the ballast force as well as the wheel-rail contact force in the presence of wheel flat utilizing comprehensive vehicle model is not available in literature. In addition to these, an in-depth investigation of the response of perfect wheel-rail contact point due to the adjacent wheel flat within the same axle is not studied. In the present paper, a dynamic computational model for the vehicle and track is formulated by means of modal analysis method. An idealized haversine wheel flat with the rounded corner is included in the wheel-rail contact model. The Rayleigh-Ritz method is employed to solve the coupled partial and ordinary differential equations of the vehicle-track system. A MATALB predefined code "ode45" has been employed to perform the time domain analyses of dynamic responses of the coupled system. The responses in terms of impact force of various vehicle and track components have been evaluated and analyzed. The effect of speed on wheel-rail impact force in the presence of single and multiple flats are investigated.

\section{VEHICLE AND TRACK SYSTEM MODEL}

The vehicle system model used in this study consists of a quarter car supported on a bogie, while the side frame is supported on two wheelsets. The primary suspension

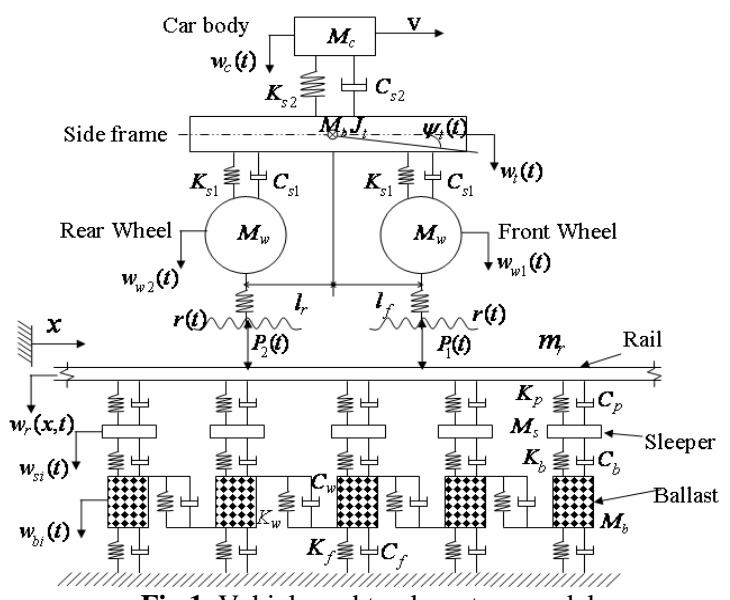

Fig.1. Vehicle and track system model. 
connecting the wheels and the bogie frame is modeled as a parallel combination of a linear spring and a viscous damping element. The secondary suspension connecting the bogie frame and the car body is also modeled by parallel spring and damping elements. The mass of the car body $M_{c}$, bogie mass $M_{t}$, wheel mass $M_{w}$ are coupled through the suspension elements, as shown in Fig. 1. The total vehicle system model is represented by a 5-DOF dynamic system that includes the car body vertical motion, $w_{c}(t)$, the bogie vertical and pitch motions, $w_{t}(t)$ and $\psi_{t}(t)$, respectively, and vertical motions of the wheels, $w_{w 1}(t)$ and $w_{w 2}(t)$. The primary suspension stiffness and damping elements are represented by $K_{s 1}$ and $C_{s 1}$ respectively, while $K_{s 2}$ and $C_{s 2}$ represent the stiffness and viscous damping coefficient due to secondary suspension. The contact force between the leading wheel and rail is denoted as $P_{1}(t)$ and that between the trailing wheel and the rail as $P_{2}(t) \cdot J_{t}$, and $r(t)$ are mass moment of inertia of the bogie, wheel defect profile. $l_{f}$ and $l_{r}$ are the distance from the mass center of the bogie to the front and rear wheel centers, respectively.

Two-dimensional model of the track system is developed upon consideration of three subsequent layers. These include the rail, and the lumped representation of the sleeper and the ballast. A finite length of the track structure is considered by including a total of 100 sleepers/ballasts. The track is assumed to be symmetric with respect to its centerline. The rail is discretely supported on the sleepers, ballasts/subballasts and subgrades elements, as shown in Fig. 1, where two layers of discrete masses below the rail represent the sleeper and the ballast, respectively. The rail beam is modeled as an Euler-Bernoulli beam and the conventional beam theory is used to analyze its deflection response under a moving load. The rotatory inertia of the beam cross section and beam deformations due to the shear force are considered negligible for the Euler beam. Railpads are placed between the steel rails and the sleepers to protect the sleepers from wear and impact damage, and they provide electrical insulation of the rails. The continuous rail beam is supported on the discrete springdamper elements representing the rail pads and fasteners. The rail is coupled to discrete sleeper masses, which provide support of the rails, and preserve level and alignment of the track through the rail pads. The ballast blocks are interconnected elastically, so that a vertical deflection of one ballast block will be distributed to others via the shear springs and dampers. It has been suggested that a total of 50 to 60 sleepers/ballast elements would be sufficient for analysis of dynamic of wheel-rail impact load due to a wheel flat ${ }^{8}$. In this study, 100 sleepers/ballasts are considered in order to investigate the contribution due to pitch dynamic of the bogie and various vehicle design parameters. The motion of the rail beam coupled with the sleeper and ballast is expressed as $w_{r}(x, t)$, while $w_{s i}(t)$ and $w_{b i}(t)$ describe the motions of the sleeper and ballast masses. $K_{p}, C_{p}, K_{b}$ and $C_{b}$ are the railpad and ballast stiffness and damping coefficients, respectively. $K_{w}$ and $C_{w}$ are the shear stiffness and damping coefficients of the ballast, respectively. The subgrade stiffness and damping are denoted as $K_{f}$ and $C_{f}$, respectively. The rail mass per unit length is represented by $m_{r}, M_{s}$ is mass of half of the sleeper, and $M_{b}$ is mass of each ballast block.

\section{SYSTEM EQUATIONS OF MOTION Equations of Vehicle}

The equations of motion of the vehicle model are derived upon neglecting the contribution due to track roughness, while the contact forces developed at the wheelrail interface are represented by $P_{1}(t)$ and $P_{2}(t)$. It is further assumed that the resultant primary suspension force acts at the bogie mass center. The equations of motion of the vehicle system are summarized below.

Car body bounce motion:

$M_{c} \ddot{w}_{c}+C_{s 2} \dot{w}_{c}+K_{s 2} w_{c}-C_{s 2} \dot{w}_{t}-K_{s 2} w_{t}=0$

Bogie bounce motion:

$M_{t} \ddot{w}_{t}+K_{s 1}\left(w_{t}+l_{f} \psi_{t}-w_{w 1}\right)+K_{s 1}\left(w_{t}-l_{r} \psi_{t}-w_{w 2}\right)+K_{s 2}\left(w_{t}-w_{c}\right)$ $+C_{s 1}\left(\dot{w}_{t}+l_{f} \dot{\psi}_{t}-\dot{w}_{w 1}\right)+C_{s 1}\left(\dot{w}_{t}-l_{r} \dot{\psi}_{t}-\dot{w}_{w 2}\right)+C_{s 2}\left(\dot{w}_{t}-\dot{w}_{c}\right)=0$

Bogie pitch motion:

$$
\begin{aligned}
& J_{t} \ddot{\psi}_{t}+K_{s 1} l_{f}\left(w_{t}+l_{f} \psi_{t}-w_{w 1}\right)-K_{s 1} l_{r}\left(w_{t}-l_{r} \psi_{t}-w_{w 2}\right)+C_{s 1} l_{f}\left(\dot{w}_{t}+l_{f} \dot{\psi}_{t}-\dot{w}_{w 1}\right) \\
& -C_{s 1} l_{r}\left(\dot{w}_{t}-l_{r} \dot{\psi}_{t}-\dot{w}_{w 2}\right)=0 \\
& M_{w} \ddot{w}_{w 1}+C_{s 1}\left(\dot{w}_{w 1}-\dot{w}_{t}\right)+K_{s 1}\left(w_{w 1}-w_{t}\right)-C_{s 1} l_{f} \dot{\psi}_{t}-K_{s 1} l_{f} \psi_{t}+P_{1}(t)=0
\end{aligned}
$$

$\underline{\text { Rear wheel vertical motion: }}$

$M_{w} \ddot{w}_{w 2}+C_{s 1}\left(\dot{w}_{w 2}-\dot{w}_{t}\right)+K_{s 1}\left(w_{w 2}-w_{t}\right)+C_{s 1} l_{r} \dot{\psi}_{t}+K_{s 1} l_{r} \psi_{t}+P_{2}(t)=0$

\section{Equations of rail track}

The equations of motion of the entire track system are derived upon integrating the equation of motion for the rail as an Euler beam with the differential equations of motions for the discrete sleeper and ballast supports. The deflection of the continuous rail can be derived from the partial differential equation for the Euler beam as:

$$
E I \frac{\partial^{4} w_{r}(x, t)}{\partial x^{4}}+m_{r} \frac{\partial^{2} w_{r}(x, t)}{\partial t^{2}}=-\sum_{i=1}^{N} F_{r i}(t) \delta\left(x-x_{i}\right)+\sum_{j=1}^{2} P_{j}^{\prime}(t) \delta\left(x-x_{j}\right)
$$

Where $N$ is total number of sleepers considered in the model, $k$ is the number of deflection modes considered for the rail beam and $j$ is the number of wheelsets incorporated in the vehicle model, which represent the number of moving point loads acting on the beam. $E$ is the elastic modulus of rail beam materials and $I$ is the second moment of area. The coordinate $X$ represents the longitudinal position of the beam with respect to the left end support of the rail beam. $X_{i}$ defines the position of the $i$ th sleeper and $\delta(x)$ is the Dirac delta function. $F_{r s i}(t)$ is force developed at the $i$ th rail/sleeper interface, given by

$$
F_{r s i}(t)=K_{p i}\left[w_{r}\left(x_{i}, t\right)-w_{s i}(t)\right]+C_{p i}\left[\dot{w}_{r}\left(x_{i}, t\right)-\dot{w}_{s i}(t)\right]
$$

The $P_{j}^{\prime}(t)$ defines the total vertical force acting at the $j$ th wheel and rail interface. It comprises both the static vehicle load and the contact force $P_{j}(t), j=1,2$, such that:

$P_{j}^{\prime}(t)=P_{j}(t)+\left[0.5\left(M_{c}+M_{t}\right)+M_{w}\right] g ; j=1,2$

The contact force $P_{j}(t)$ is derived using the Hertzian contact model described in Eq. (11), such that: 
$P_{j}(t)=C_{H}\left[w_{w j}(t)-w_{r}\left(x_{j}, t\right)-r_{j}(t)\right]^{3 / 2} ; j=1,2$

Where $C_{H}$ is Hertzian wheel/rail contact coefficient and $r_{j}(t)$ is the wheel flat function.

The contact force diminishes when a loss of contact of the wheel with the rail is encountered, when $\left[w_{w j}(t)-w_{r}\left(x_{j}, t\right)-r_{j}(t)\right] \leq 0 ; j=1,2$

The rail beam deflection $w_{r}(x, t)$ is evaluated through solution of Eq. (2.6) using the generalized coordinates method. The deflection $w_{r}(x, t)$ is expressed by the product of the $k$ th mode deflection mode $Y_{k}(x)$ and the time coordinate $q_{k}(t)$. The equation of motion for the discrete sleeper and ballast masses are derived as follows:

$$
\begin{array}{r}
M_{s i} \ddot{w}_{s i}(t)+\left(C_{p}+C_{b}\right) \dot{w}_{s i}(t)+\left(K_{p}+K_{b}\right) w_{s i}(t)-C_{b} \dot{w}_{b i}(t)-K_{b} w_{s i}(t) \\
-C_{p} \sum_{k=1}^{K} Y_{k}\left(x_{i}\right) \dot{q}_{k}(t)-K_{p} \sum_{k=1}^{K} Y_{k}\left(x_{i}\right) q_{k}(t)=0 ; \quad i=1,2, \ldots . . N
\end{array}
$$$$
M_{b i} \ddot{w}_{b i}(t)+\left(C_{b}+C_{f}+2 C_{w}\right) \dot{w}_{b i}(t)+\left(K_{b}+K_{f}+2 K_{w}\right) w_{b i}(t)-C_{b} \dot{w}_{s i}(t)-K_{b} w_{s i}(t)
$$

$$
\begin{gathered}
-C_{w} \dot{w}_{b(i+1)}(t)-K_{w} w_{b(i+1)}(t)-C_{w} \dot{w}_{b(i-1)}(t)-K_{w} w_{b(i-1)}(t)=0 ; \\
i=1,2, \ldots . . N
\end{gathered}
$$

The shear coupling between the first ballast mass and the beam support, and the last ballast mass and the beam support, however, are not considered in Eq. (9), i.e. $w_{b 0}(t)=\dot{w}_{b 0}=0$; and $w_{b(N+1)}(t)=\dot{w}_{b(N+1)}(t)=0$.

The ballast force developed at the sleeper-ballast interface can be expressed as:

$F_{s i i}(t)=K_{b i}\left[w_{s i}(t)-w_{b i}(t)\right]+C_{b i}\left[\dot{w}_{s i}(t)-\dot{w}_{b i}(t)\right]+K_{w i}\left(w_{b i}-w_{b+i}\right)+C_{w i}\left(\dot{w}_{b i}-\dot{w}_{b+i}\right)$

$$
+K_{w i}\left(w_{b i}-w_{b-i}\right)+C_{w i}\left(\dot{w}_{b i}-\dot{w}_{b-i}\right)
$$

\section{Wheel-rail interaction}

Wheel-rail interface distinguishes railways from other forms of land transports. In order to simulate the wheel-rail interaction force, the contact between the wheel and rail must be established by a well-defined method, since an accurate solution of the rolling contact problem is quite complex. The essential characteristic of the wheel-rail contact is its extreme stiffness in vertical direction. The area of contact is generally very small, while the interface supports the traction, braking, and curving forces apart from the vertical forces.

The interaction between the vehicle and the track system is achieved at the wheel/rail interfaces through wheel/rail force compatibility. The wheel-rail contact has been widely described by the nonlinear Hertzian contact theory commonly used in the wheel/rail interaction problems ${ }^{1,6,8}$. According to the Hertzian contact theory, the wheel-rail contact force is related to the rail deflection in a nonlinear manner, such as:

$$
P(t)=C_{H} \Delta z(t)^{3 / 2}
$$

Where $\Delta z(t)$ is the wheel-rail overlap in the vertical direction. In the absence of a wheel defect, the overlap is defined by the relative motion of the wheel with respect to the rail:

$$
\Delta z(t)=w_{w}(t)-w_{r}(x, t)-r(t)
$$

Where $r(t)$ is the wheel flat function. $w_{w}(t)$ and $w_{r}(x, t)$ are the wheel and rail deflections in vertical direction, respectively.

A haversine flat is expressed as:

$$
r=\frac{1}{2} D_{f}\left[1-\cos \left(2 \pi x / L_{f}\right)\right]
$$

Where $D_{f}$ is the flat depth, $L_{f}$ is the length of the flat, $X$ is the longitudinal coordinate of the contact point within the flat.

\section{ANALYSIS METHOD}

The track system model formulated in this study comprises both ODEs and PDE describing the deflection of the lumped sleeper and ballast masses, and the continuous rail, respectively. The PDE is expressed as ODEs by assuming a mode shape function. The Rayleigh-Ritz method is used to express the fourth order PDE describing the motion of the continuous rail by a series of second order ordinary differential equations in terms of the time coordinates. The resulting ODEs of the track and vehicle systems are than solved in time domain to obtained responses of individual components of the vehicle-track system model. The relative responses between the components are used to derive the dynamic interaction forces. The closed form of the solutions by using this method largely depends on the accurate assumption of the mode shape and the number of modes considered. In this study, a total of 100 modes of the rail beam are considered for analysis of the coupled vehicle-track system model.

The deflection mode of continuous beam with simply supported boundary conditions can be derived from the Euler-Bernoulli equation of the beam. The deflection modes and the natural frequencies of an Euler beam inn the absence of sleeper supports and external loads have been well documented and can be expressed as ${ }^{6}$ :

$$
\begin{aligned}
& Y_{k}(x)= \sin \left(\frac{k \pi x}{l}\right) ; \text { and } \\
& \omega_{k}=\left(\frac{k \pi}{l}\right)^{2} \sqrt{\frac{E I}{m_{r}}}, k=1,2,3 \ldots \ldots \ldots K
\end{aligned}
$$

Where $Y_{k}(x)$ is the deflection mode, $\omega_{k}$ is the corresponding natural frequency and $l$ is the beam length. The deflection response of the rail is then derived from :

$$
w_{r}(x, t)=\sum_{k=1}^{K} Y_{k}(x) q_{k}(t)
$$

Where $K$ is the number of modes considered.

The rail deflections in the vicinity of the contact points with the wheels are derived from:

$$
\begin{aligned}
& w_{r}\left(x_{1}, t\right)=\sum_{k=1}^{K} Y_{k} \sin \left(\frac{k \pi}{l} v t\right) q_{k}(t) ; \text { and } \\
& \quad w_{r}\left(x_{2}, t\right)=\sum_{k=1}^{K} Y_{k} \sin \left[\frac{k \pi}{l}\left(v t-l_{t}\right)\right] q_{k}(t)
\end{aligned}
$$

Where $l_{t}=l_{f}+l_{r}$ is the wheel base of the bogie.

The substitution of the rail deflection response from Eq. (15) together with the mode shape $Y_{k}$ in the PDE, Eq. (6) yields a set of ODEs in $q(t)$, expressed as: 


$$
\begin{array}{r}
\ddot{q}_{k}(t)+\alpha \sum_{i=1}^{N} C_{p i} Y_{k}\left(x_{i}\right) \sum_{k=1}^{K} Y_{k}\left(x_{i}\right) \dot{q}_{k}(t)+\frac{E I}{m_{r}}\left(\frac{k \pi}{l}\right)^{4} q_{k}(t)+\alpha \sum_{i=1}^{N} K_{p i} Y_{k}\left(x_{i}\right) \sum_{k=1}^{K} Y_{k}\left(x_{i}\right) q_{k}(t) \\
-\alpha \sum_{i=1}^{N} C_{p i} Y_{k}\left(x_{i}\right) \dot{w}_{s i}(t)-\alpha \sum_{i=1}^{N} K_{p i} Y_{k}\left(x_{i}\right) w_{s i}(t)=\alpha \sum_{j=1}^{2} P_{j}^{\prime}(t) Y_{k}\left(x_{G j}\right)
\end{array}
$$

Where $\alpha=\left(2 / m_{r} l\right)$ and $k=1,2,3 \ldots \ldots \ldots K$

The above equations incorporate the effects of sleeper supports through the restoring and dissipative forces developed by the rail pad in the vicinity of the sleeper support. The total force of the rail pad, attributed to rail deflections alone, can be further simplified as:

$$
\begin{aligned}
& \sum_{i=1}^{N} K_{p i} Y_{k}\left(x_{i}\right) \sum_{k=1}^{K} Y_{k}\left(x_{i}\right) q_{k}(t)+\sum_{i=1}^{N} C_{p i} Y_{k}\left(x_{i}\right) \sum_{k=1}^{K} Y_{k}\left(x_{i}\right) \dot{q}_{k}(t)= \\
& K_{p i} \sum_{i=1}^{N} \sum_{k=1}^{K} Y_{k}\left(x_{i}\right) Y_{k}\left(x_{i}\right) q_{k}(t)+C_{p i} \sum_{i=1}^{N} \sum_{k=1}^{K} Y_{k}\left(x_{i}\right) Y_{k}\left(x_{i}\right) \dot{q}_{k}(t)
\end{aligned}
$$

As example, these forces associated with the $K$ th mode ( $k=K$ ) can be derived from:

$$
\begin{aligned}
& K_{p i} \sum_{i=1}^{N} Y_{k}\left(x_{i}\right) Y_{k}\left(x_{i}\right) q_{k}(t)+C_{p i} \sum_{i=1}^{N} Y_{k}\left(x_{i}\right) Y_{k}\left(x_{i}\right) \dot{q}_{k}(t)= \\
& K_{p} q_{K}\left[Y_{K}\left(x_{1}\right) Y_{K}\left(x_{1}\right)+Y_{K}\left(x_{2}\right) Y_{K}\left(x_{2}\right)+Y_{K}\left(x_{3}\right) Y_{K}\left(x_{3}\right)+\ldots \ldots \ldots+Y_{K}\left(x_{N}\right) Y_{K}\left(x_{N}\right)\right] \\
&+\quad \\
& \quad C_{p} \dot{q}_{K}\left[Y_{K}\left(x_{1}\right) Y_{K}\left(x_{1}\right)+Y_{K}\left(x_{2}\right) Y_{K}\left(x_{2}\right)+Y_{K}\left(x_{3}\right) Y_{K}\left(x_{3}\right)+\ldots \ldots . .+Y_{K}\left(x_{N}\right) Y_{K}\left(x_{N}\right)\right]
\end{aligned}
$$

The equation of motion of the rail supported on discrete sleepers and railpads are formulated upon substituting for component of the railpad force, described above, in Eq. (17). These equations are summarized below, as example,

For, $k=100$

$$
\begin{aligned}
& \ddot{q}_{100}(t)+\alpha \sum_{i=1}^{N} C_{p i} Y_{100}\left(x_{i}\right) Y_{100}\left(x_{i}\right) \dot{q}_{100}(t)+\frac{E I}{m_{r}}\left(\frac{100 \pi}{l}\right)^{4} q_{100}(t)+\alpha \sum_{i=1}^{N} K_{p i} Y_{100}\left(x_{i}\right) Y_{100}\left(x_{i}\right) q_{100}(t) \\
& -\alpha \sum_{i=1}^{N} C_{p i} Y_{100}\left(x_{i}\right) \dot{w}_{s i}(t)-\alpha \sum_{i=1}^{N} K_{p i} Y_{100}\left(x_{i}\right) w_{s i}(t)=\alpha \sum_{j=1}^{2} P_{j}^{\prime}(t) Y_{k}\left(x_{G j}\right)
\end{aligned}
$$

The deflection response of the rail is finally derived from summation of a total of 100 deflection modes, as:

$w_{r}(x, t)=Y_{1}(x) q_{1}(t)+Y_{2}(x) q_{2}(t)+Y_{3}(x) q_{3}(t)+$ $\ldots .+Y_{100}(x) q_{100}(t)$

The equations of motion of the vehicle system described by Eqs. (1) to (5), and of the track system derived in Eqs. (6) to (9) and (17) together with the Hertzian nonlinear contact model in Eq. (11) describe the vertical dynamics of the coupled vehicle-track system. Detail derivation of the vehicle-track model and method of analysis can be found in ${ }^{11}$.

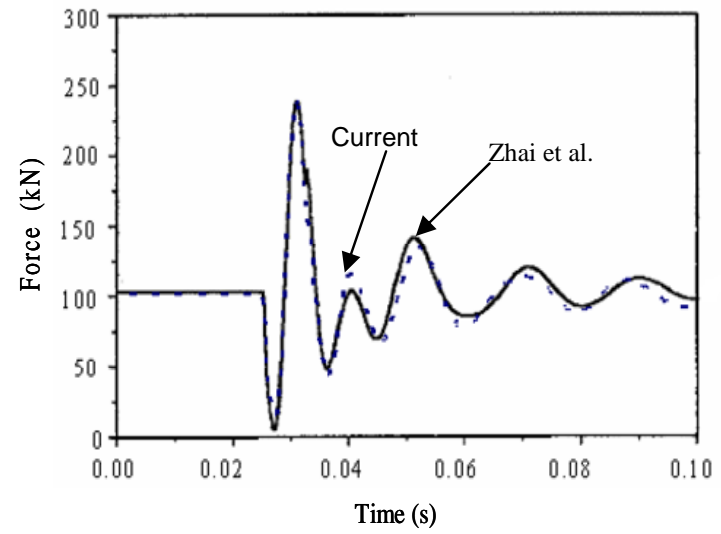

Fig. 2: Comparison of wheel-rail impact force response of the present model with that reported by Zhai et al. ${ }^{6}$.

\section{RESPONSE ANALYSES OF THE VEHICLE-TRACK SYSTEM}

The developed model is validated using the data reported by Zhai et al. ${ }^{6}$. In this reported study, a 10-DOF pitch plane model of the full car was considered with a three-layer model. The rail was modeled as an EulerBernoulli beam. All the parameters of the vehicle, the track, and defect size considered in this validation are taken from Zhai et al. ${ }^{6}$, except for the primary suspension properties and Hertzian contact stiffness, which were not reported. These parameters were obtained for a typical wagon track system used in North America. The dynamic response of the entire vehicle-track system is evaluated under a constant speed of $27 \mathrm{~km} / \mathrm{h}$ as reported by Zhai et al. $^{6}$ in the presence of a $52.8 \mathrm{~mm}$ long and $1 \mathrm{~mm}$ deep flat in the leading wheel. The variation in the dynamic contact force obtained at the interface of the defective wheel and rail is evaluated and compared with those reported in ${ }^{6}$, as shown in Fig. 2. The simulation results were obtained under steady-state condition.

It can be seen that the contact force response predicted by the current model agrees reasonably well with the response reported by Zhai et al. ${ }^{6}$. It should be noted that the reported study employed a 10 DOF pitch plane model of the entire car, while the present study considers only a 5DOF model of the quarter car. Despite this simplification, the current model yields nearly similar impact force response, which suggests negligible contributions due to the vehicle pitch. A reduced model would thus be sufficient for accurately predicting the dynamic contact force due to a wheel flat. The amplitude of impact force and the period of vibrations predicted by both models are in very good agreement. The peak impact force predicted by both models is in the order of $245 \mathrm{kN}$. The dominant period of oscillation of the contact force is approximately $0.01 \mathrm{~s}$ for both models. The results attained from both the models suggest that this period of oscillation rapidly approaches to nearly $0.02 \mathrm{~s}$ after the excitation due to wheel flat has passed the contact region, although some differences in the responses become evident.

Figure 3 (a) further illustrates the fron(21)heel-rail contact force response of the present model over a longer duration for examining the possible response when the excitation due to the flat is absent. The ratio of peak force to the static load is nearby 2.37. Figure 3 (b) shows the contact force response at flat-free rear wheel-rail contact point. The results clearly show significantly high contact force at the rear wheel-rail interface, even though the rear wheel is considered to be free of defects. The peak impact force caused by the rear wheel flat approaches $166.4 \mathrm{kN}$. The ratio of peak force to the static load is nearby 1.62 . Unlike the impact force response at the front wheel contact, the impact force of the rear wheel exhibits a smaller magnitude peak prior to the sharp drop in contact force, which can be attributed to out-of-phase motion of the two wheel-rail contact points. The oscillations in the contact force of the rear wheel tend to diminished over a short duration of approximately $0.04 \mathrm{~s}$. The wheel-rail contact force, however, continues to oscillate at a frequency near $56 \mathrm{~Hz}$ until an impact of the front wheel flat occurs during the subsequent cycle. The impact force developed at the perfect wheel-rail contact due to a flat in another wheel is also referred to as the 'cross-wheel' force, and has been attributed to side frame pitch ${ }^{12}$.

The results in Fig. 3 further show the sequence of events in terms of contact force and their frequency of oscillation, which can be explained in the following 


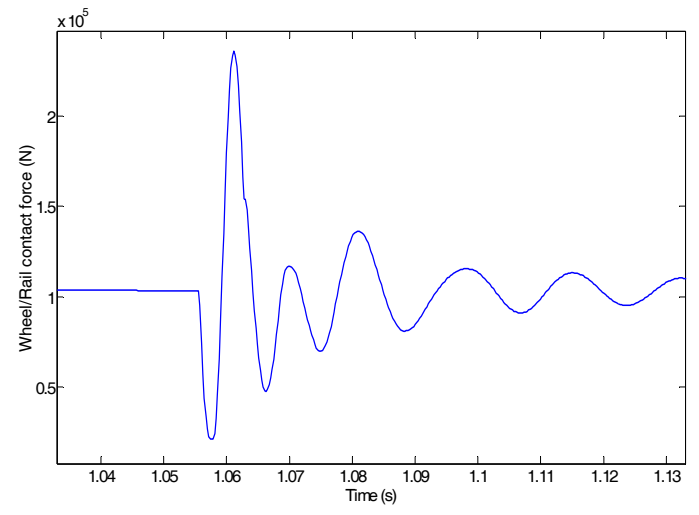

(a) front wheel-rail impact with flat

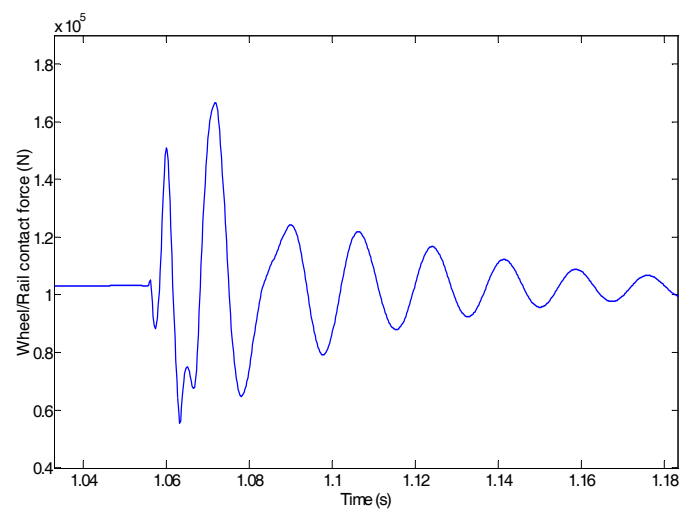

(b) flat-free rear wheel-rail impact

Fig. 3: Time-history of impact force response predicted by the current model: (a) front wheel-rail impact with flat; and (b) flat-free rear wheel-rail impact ( $\mathrm{v}=27$ $\mathrm{km} / \mathrm{h} ; L_{f}=52.8 \mathrm{~mm} ; D_{f}=1 \mathrm{~mm}$ )

manner. As the wheel flat enters the contact area, there is a sudden drop in the contact force followed by a large peak due to the wheel-rail impact. This referred to as P1 force in literature ${ }^{6}$, lasts for a duration of $0.008 \mathrm{~s}$, which correspond to the duration of the flat in contact with the rail. The frequency of P1 can therefore be referred to as the excitation frequency due to the flat, and is a function of flat size and forward speed. The following sequence of peak force known as P2 force oscillates at a frequency of 125 $\mathrm{Hz}$. This is primarily due to oscillation of the rail on the support pads.

The validated vehicle-track system model is applied to investigate the dynamic contact force as well as dynamic responses of the components at a constant forward speed. The analyses are performed under a haversine wheel flat ( $L_{f}=52 \mathrm{~mm}$ and $D_{f}=0.4 \mathrm{~mm}$ ). The type of flat considered closely meets the wheel removal criterion recommended by a number of American and European railroad organizations, such as $\mathrm{AAR}^{13}$, Transport Canada ${ }^{14}$, Swedish Railway ${ }^{2}$, and UK Rail Safety and Standard Board $^{15}$.

The equations of motion for the vehicle-track model are solved for the track length involving 100 sleepers, and a forward speed $70 \mathrm{~km} / \mathrm{h}$. The simulations were performed were performed using a time step size of $0.000078 \mathrm{~s}$, which is significantly smaller than the time required for the flat to
Table 1: Nominal simulation parameters

\begin{tabular}{lll}
\hline Sym. & Parameter & Value \\
$M_{c}$ & Car body mass (quarter car) & $19400 \mathrm{~kg}$ \\
$M_{t}$ & Bogie mass (half) & $500 \mathrm{~kg}$ \\
$M_{w}$ & Wheel mass & $500 \mathrm{~kg}$ \\
$J_{t}$ & Bogie mass moment inertia & $176 \mathrm{~kg}-\mathrm{m}^{2}$ \\
$K_{s 1}$ & Primary suspension stiffness & $788 \mathrm{MN} / \mathrm{m}$ \\
$C_{s 1}$ & Primary suspension damping & $3.5 \mathrm{kN}-\mathrm{s} / \mathrm{m}$ \\
$K_{s 2}$ & Secondary suspension stiffness & $6.11 \mathrm{MN} / \mathrm{m}$ \\
$C_{s 2}$ & Secondary suspension damping & $158 \mathrm{kN}-\mathrm{s} / \mathrm{m}$ \\
$l_{t}$ & Wheelset distance & $1.25 \mathrm{~m}$ \\
$R$ & Wheel radius & $0.42 \mathrm{~m}$ \\
$L_{f}$ & Flat length & $52 \mathrm{~mm}$ \\
$D_{f}$ & Flat depth & $0.4 \mathrm{~mm}$ \\
$C_{H}$ & Hertzian spring constant & $87 \mathrm{GN} / \mathrm{m}^{3 / 2}$ \\
$m_{r}$ & Rail mass per unit length & $60.64 \mathrm{~kg} / \mathrm{m}$ \\
$E I$ & Rail bending stiffness & $6.62 \mathrm{MN}-\mathrm{m}^{2}$ \\
$M_{s}$ & Sleeper mass & $118.5 \mathrm{~kg}$ \\
$M_{b}$ & Ballast mass & $739 \mathrm{~kg}$ \\
$K_{p}$ & Railpad stiffness & $120 \mathrm{MN} / \mathrm{m}$ \\
$K_{b}$ & Ballast stiffness & $182 \mathrm{MN} / \mathrm{m}$ \\
$K_{w}$ & Ballast shear stiffness & $147 \mathrm{MN} / \mathrm{m}$ \\
$K_{f}$ & Subgrade stiffness & $78.4 \mathrm{MN} / \mathrm{m}$ \\
$C_{p}$ & Railpad damping & $75 \mathrm{kN}-\mathrm{s} / \mathrm{m}$ \\
$C_{b}$ & Ballast damping & $58.8 \mathrm{kN}-\mathrm{s} / \mathrm{m}$ \\
$C_{w}$ & Ballast shear damping & $80 \mathrm{kN}-\mathrm{s} / \mathrm{m}$ \\
$C_{f}$ & Subgrade damping & $31.15 \mathrm{kN}-\mathrm{s} / \mathrm{m}$ \\
$l_{s}$ & Sleeper distance & $0.6 \mathrm{~m}$ \\
$N$ & No. of sleepers & 100 \\
\hline & &
\end{tabular}

overcome the contact with the rail. The simulation parameters used for analysis are given in the Table 1, which represent those of a typical North American railroad car.

The high magnitude contact forces developed at the wheel-rail interface are transmitted to the vehicle components and track layers, which may cause components fatigue or failure. The transmission of contact force to the sideframe occurs through the bearing adapters. The deflections of wheel and side frame give rise to the primary suspension force, also referred to as bearing force, which is eventually transmitted to the car body. The magnitudes of bearing forces strongly depend upon the nature of flat, speed, wheel load and suspension properties. The variations in bearing force are investigated using the model parameters listed in Table 1 and a flat within the rear wheel $\left(L_{f}=52 \mathrm{~mm} ; D_{f}=0.4 \mathrm{~mm}\right)$. The responses, evaluated under three different load conditions $(\mathrm{W}=63$, 82, and $102 \mathrm{kN}$ ), are compared in Fig. 4. The bearing force tends to rapidly decrease from its static level of $100 \mathrm{kN}$ as the wheel flat enters the wheel-rail contact region, as it was observed for the wheel-rail contact force in Fig. 3. Similar to the contact force, the bearing force increases to a high value of nearly $160 \mathrm{kN}$, although the rate of rise is considerably smaller than that of the contact force. The bearing force decays in an exponential manner, thereafter, which is attributed to the primary suspension damping.

The results further show that variations in the wheel load do not influence the oscillation frequency of the bearing force. The ratio of peak bearing force to static bearing force varies nonlinearly with the wheel load. These ratios for wheel loads of 102,82 and $63 \mathrm{kN}$ are obtained as $1.59,1.73,1.93$, respectively. The nonlinear variations in the peak bearing force with the wheel load are mostly attributed to the nonlinear variations in the peak wheel-rail impact force with the static wheel load, which is further 


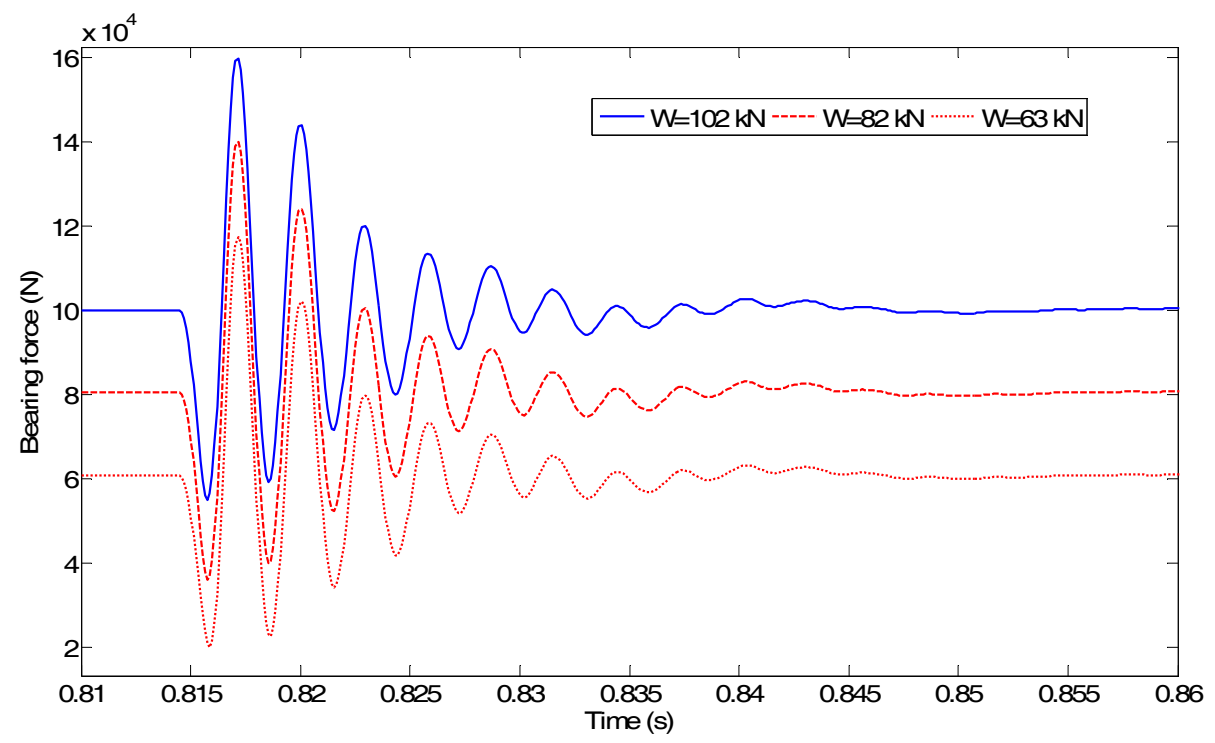

Fig. 4: Variations in the bearing force response due to a rear-wheel flat as a function of static wheel load.

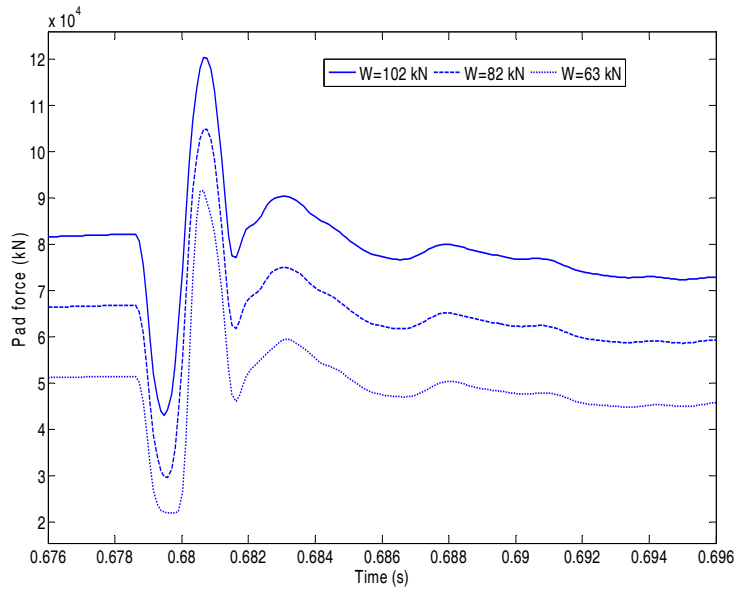

Fig. 5: Variations in railpad force due to a rear wheel flat as a function of static wheel load.

attributed to the nonlinear wheel-rail contact model. The ratios of peak wheel-rail impact load to static wheel load were obtained as 1.77, 1.96, and 2.45 for static loads of 102, 82 and $63 \mathrm{kN}$, respectively.

The dynamic force developed by the discrete rail pads, also referred to as the reaction force at each railsleeper interface, is further evaluated to study the nature of forces transmitted to different track layers. The dynamic rail pad force is computed from relative deflection of the rail and sleeper, using Eq. (7). As an example, the variations in the rail pad force developed at sleeper no. 22, due to a flat within the rear wheel, are illustrated in Fig. 5. The variations in the railpad force are investigated using the model parameters listed in Table 1 and a flat within the rear wheel ( $L_{f}=52 \mathrm{~mm} ; D_{f}=0.4 \mathrm{~mm}$ ), and three different load conditions (W=63, 82, $102 \mathrm{kN}$ ) at a constant forward vehicle speed of $70 \mathrm{~km} / \mathrm{h}$. Figure 5 shows that as the wheel flat enters the wheel-rail contact region, the pad force rapidly decreases from its static level of 82.23, 66.88, and $51.57 \mathrm{kN}$ under static wheel loads of 102, 82 and $63 \mathrm{kN}$, respectively. The pad force then increases to peak value of

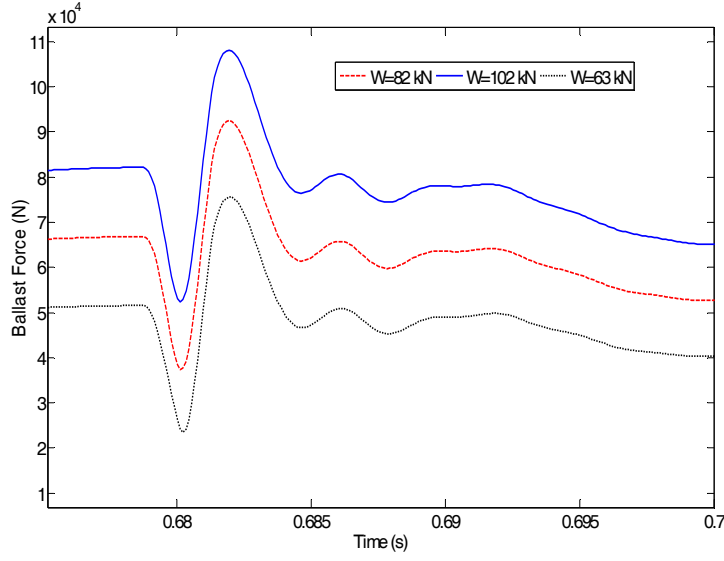

Fig. 6: Variations in ballast force due to a rear wheel flat as a function of static wheel load.

$120.4,104.9$, and $91.64 \mathrm{kN}$, respectively. The ratios of the peak pad force to static pad force are obtained as 1.46 , 1.57, and 1.77 for static wheel load of 102, 82 and $63 \mathrm{kN}$, respectively. The figure further shows that the pad force gradually decreases after the impact, as the wheel moves away from the location of the sleeper. It can be seen that the variations in the wheel load do not influence the oscillation frequency of the pad force. The results also show that the magnitudes of pad forces are significantly smaller than those of the contact forces, which can be attributed to inertia force of the rail.

The impact force developed at wheel-rail interface due to a wheel flat is also transmitted to the ballast blocks, which is ultimately transmitted to the ground. The force developed at the sleeper-ballast interface is known as ballast force. The magnitudes of ballast forces also depend upon the wheel load, ballast properties and the nature of the flat. The effect of wheel flat on dynamic ballast force is investigated in the presence of a single flat in the rear wheel. The resulting variations in the ballast force are shown in Fig. 6. The ballast force is computed by summing up the reaction forces obtained from a particular sleeper and the shear forces arising from couplings with the 
adjacent ballast blocks, as shown in Eq. (10). The time histories of the ballast forces for ballast no. 22 under three different load conditions ( $\mathrm{W}=63,82$ and $102 \mathrm{kN}$ ) are shown in Fig. 6. The results suggest that variations in ballast forces are similar to those observed for the pad forces shown in Fig. 5. The peak magnitudes of the ballast forces, however, are considerably smaller than those of the pad forces. The peak values of forces are attained as 108.4 , 92.49 and $75.62 \mathrm{kN}$ under static wheel loads of 102, 82 and $63 \mathrm{kN}$, respectively. The ratios of peak to static ballast forces for wheel loads of 102, 82, and $63 \mathrm{kN}$ are obtained as $1.32,1.38$, and 1.46, respectively. The normalized ballast forces relatively less sensitive to variations in the static wheel load in the range of variations considered. The figure further shows that the ballast force gradually decreases after the wheel impact, as the wheel moves away from the location of the ballast. The variations in the wheel loads do not show significant influence on the oscillation frequency of the ballast force, as observed for the wheelrail contact and bearing forces.

The deflection responses of different vehicle and track system components are further investigated to study the transmission of wheel flat-induced rail deflections to various vehicle components and track layers, as a vehicle moves over the track with a particular load and speed. Excessive deflections may cause fatigue damage of the vehicle-track components, especially the track layers leading to increase maintenance cost or track failure. The deflections of the vehicle and track components are evaluated under constant static wheel load of $102 \mathrm{kN}$ in the presence of a rear wheel flat $\left(L_{f}=52 \mathrm{~mm} ; D_{f}=0.4 \mathrm{~mm}\right)$.

Figure 7 and 8 illustrate the variations in displacements responses of wheel-rail contact points at front and rear wheels, respectively. The results show deflections of the wheel masses and deflections of rail at wheel-rail contact points. The application of the static wheel load causes the rail to deform downwards at the contact point. However, a relaxation of rail compression prior to the impact at the contact point is evident as the flat approaches the contact region, while the wheel moves downwards due to its flat geometry. This is followed by further compression of the rail due to impact force developed at the wheel-rail interface, as seen in Fig. 7. Subsequently, the rail profile tends to recover its steady value, while the deflection response exhibits oscillation near $56 \mathrm{~Hz}$, which has been referred to as the coupled

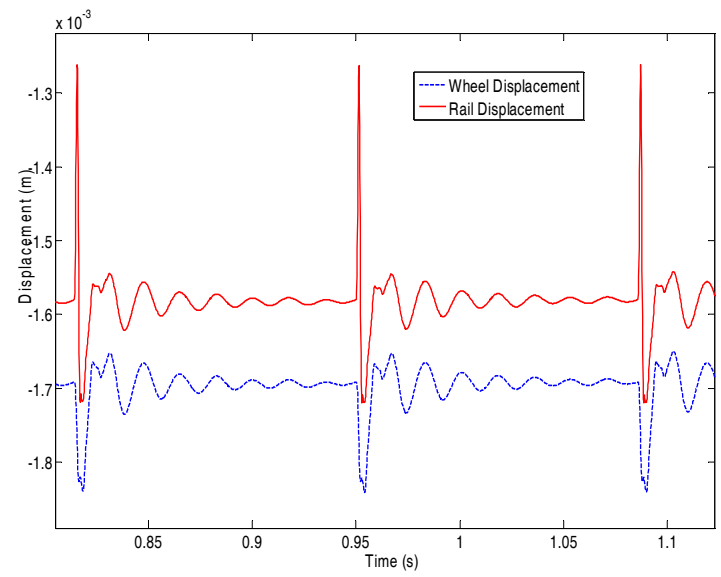

Fig. 7: Time histories of vertical displacements of the rear wheel and rail in the presence of a flat

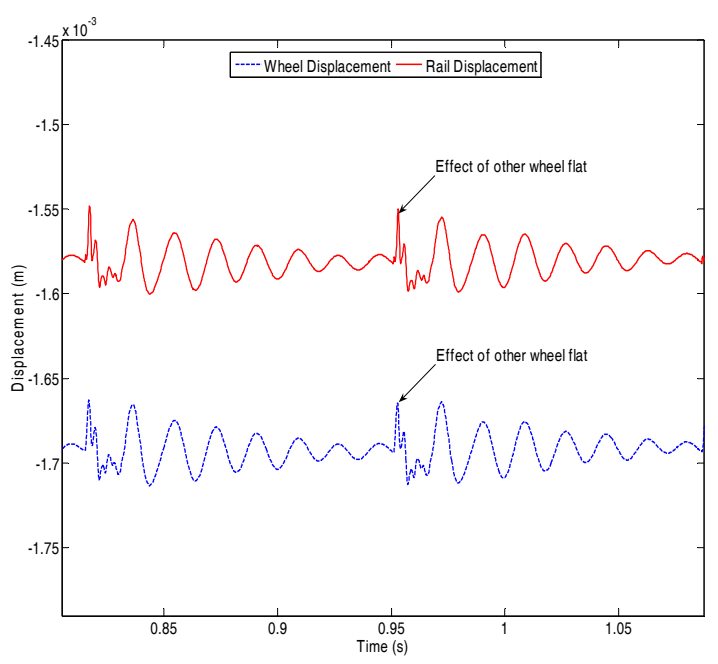

Fig. 8: Time histories of vertical displacements of front wheel (no flat) and rail at the wheel-rail contact point.

vehicle-track system resonance ${ }^{2,16}$. Both the wheel and rail displacement responses exhibit oscillation at the same frequency.

The impact forces caused by the rear wheel flat also yields deflections of flat-free front wheel and the corresponding wheel-rail contact point, as shown in Fig. 8, although the peak deflections are relatively small. This is attributed to the influence of the cross wheel impact force. The results further show that peaks in deflections responses of the wheel and the rail occurs at same instants. Furthermore, in the absence of flat, the difference between the displacements of the wheel and the rail remains nearly constant over the entire revolution of the wheel.

Fig. 9 (a) illustrates the variations in displacement response at a single point on the rail while flat $\left(L_{f}=52\right.$ $\mathrm{mm} ; D_{f}=0.4 \mathrm{~mm}$ ) is assumed to exist in the rear wheel.

Figures 9 (b) and (c) illustrate the resulting variations in displacement responses of the sleeper and ballast block ( $i=$ 22) located beneath the point considered on the rail. The results clearly show the differences between peak deflections of the point on the rail, sleeper and ballast under the front and rear wheel contacts. The point on the rail undergoes a peak deflection of $1.58 \mathrm{~mm}$ under the front wheel contact, which increases to $1.715 \mathrm{~mm}$ under the rear wheel. Similarly, the peak deflections of the sleeper under front and rear wheel contacts are obtained as $0.88 \mathrm{~mm}$ and $0.99 \mathrm{~mm}$, respectively, while those of the ballast are 0.46 $\mathrm{mm}$ and $0.5 \mathrm{~mm}$. While the deflection response at the front wheel-rail contact point is affected by the rear-wheel flat, the effect is not evident in Fig. 9 (a) due to the relatively large ordinate scale. This effect, however, is evident in the ballast response as shown in Fig. 9 (c). The rail, sleeper, and ballast deflections exhibit small fluctuations every time as the deflections approach their steady values. These are attributed to impact forces occurring at adjacent locations of the rail. The results also show slight rail lift-off from the sleeper, sleeper lift-off from the ballast and ballast lift-off from the sub-grade occur in the order of $0.052 \mathrm{~mm}, 0.0229$ $\mathrm{mm}$, and $0.0073 \mathrm{~mm}$, respectively. These lift-offs can be attributed to the dynamics of the moving load that separate the track components from each other.

The presence of a wheel flat, however, significantly influences both the bounce and pitch deflection responses 

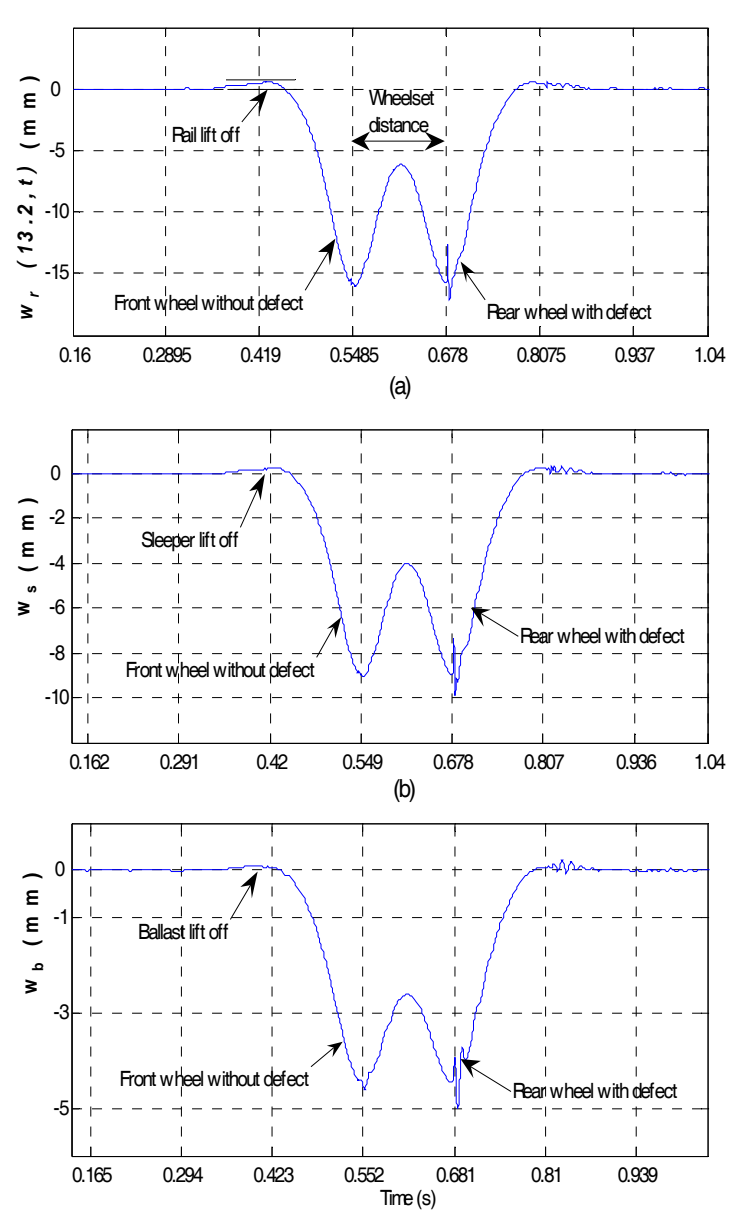

Fig. 9: Displacement responses(\&valuated at (a) a point on the rail; (b) sleeper; and (c) ballast beneath the rail point in the presence of a rear wheel flat

of the bogie, as shown in Fig. 10. Both the vertical and pitch deflections of the bogie increase rapidly as the flat interacts with the rail. This is caused by relatively high magnitude bearing forces transmitted to the side frame, as observed in Fig. 4. As the flat moves away, the deflection responses oscillate around the static equilibrium near the frequency of $56 \mathrm{~Hz}$. In the presence of a flat, the peak vertical deflection of the bogie increases from the static value of $1.816 \mathrm{~mm}$ to $1.904 \mathrm{~mm}$. A rapid change in the pitch response of the bogie also occurs at the time when the defective wheel strikes the rail. The peak pitch angle, however, is relatively small, in the order of $0.8 \times 10^{-6} \mathrm{rad}$. The results suggest important effects of wheel flat on the vertical deflection of the bogie mass, which would be further influenced by an array of factors related to operating variables and flat geometry.

\section{DISCUSSION}

The characteristic of the impact load due to a haversine wheel flat is investigated by a pitch plane vehicle model. The influence of the flat on various components of the vehicle and track is analyzed. This study illustrates that wheel flat has enormous influence on all the components of vehicle and track system except the car body. The current study also shows that flat present in one wheel has strong influence not only in contact forces but also in the

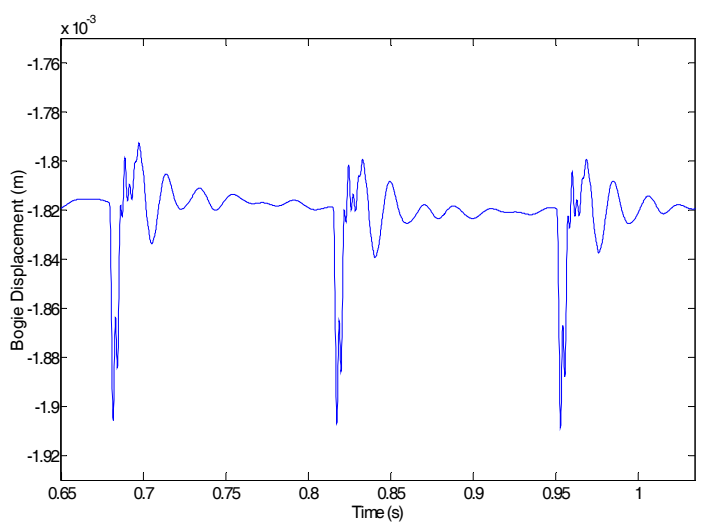

(a) bounce motion(a) bounce motion

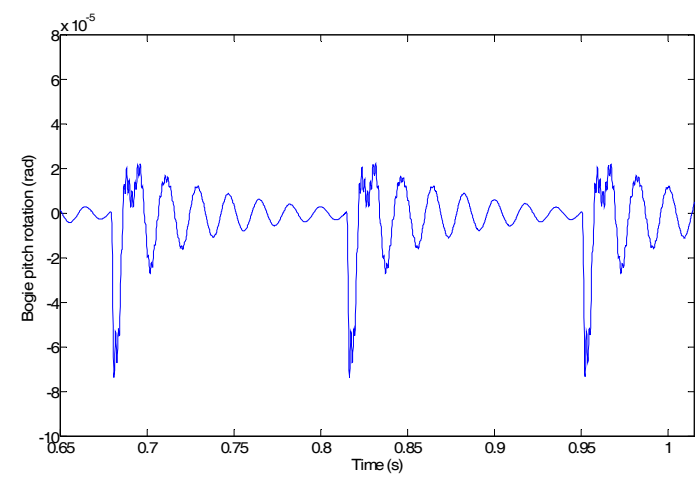

(b) pitch motion.

Fig. 10: Variation in the bounce and pitch responses of the bogie in the presence of a rearwheel flat $\left(L_{f}=52\right.$ $\mathrm{mm} ; D_{f}=0.4 \mathrm{~mm}$ ): (a) bounce motion; (b) pitch motion.

displacements responses. The investigations of the wheelrail impact loads, bearing, railpad, and ballast forces due to a haversine wheel flat as a function of the static wheel load show that the magnitudes of these transmitted forces increase considerably under a flat-induced excitation, which may cause rapid fatigue of the vehicle and track components. The magnitudes of these impact forces could be approaches twice the static load under medium wheel loads. The results also suggested significant influences of the wheel flat on displacement responses of the vehicle and track system components except for the low natural frequency car body. The study also revealed that a flat in one of the bogie wheels would also influence the forces developed at the adjacent wheel-rail contact point. A defective wheel may thus cause damage to the wheels of the adjacent wheelset. The magnitude of this cross-wheel force depends on the vehicle, track properties, and the flat geometry. It is shown that a flat in a wheel not only affects the wheel-rail deflections but also the deflections of the sleepers and the ballast. Furthermore, a certain amount of sleepers, and ballasts lift-off occur due to the dynamics of vehicle's moving load that might separate the track components from each other. This amount of lift-off can vary depending on the speed and positions of the loads, sleepers, and ballasts. 


\section{REFERENCES}

1. Thompson, D., and Wu, T.X., "The effect of nonlinearity on wheel/rail impact" Journal of Rail and Rapid transit, volume 218, part F, 2003, p1-15.

2. Sun, Y. Q., Dhanasekar, M., and Roach, D., "A threedimensional model for the lateral and Vertical Dynamics of wagon-track system.” Proceedings of the institution of Mechanical Engineers, Part F: Rail and Rapid Transit. Vol. 217 (1), 2003, p 31-45.

3. Hou, K., Kalousek, J., Dong, R., “A dynamic model for an asymmetrical vehicle/track system" Journal of Sound and Vibration 267 (2003) 591-604.

4. Knothe, K., and Grassie, S.L., "Modeling of railway track and vehicle/track interaction at high frequencies", Vehicle System Dynamics 22 1993, pp. 209 - 262.

5. Wen, Z., Jin, X., and Zhang, W., "Contact-impact stress analysis of rail joint region using the dynamic finite element method” Wear 258 (2005) p1301-1309.

6. Zhai, W. M., Cai, C. B., Wang, Q. C., Lu, Z.W., and Wu., X. S., "Dynamic Effects of Vehicles on Tracks in the Case of Raising Train Speed" Proceedings of the Institution of Mechanical Engineers, Part F, v 215, 2001, p125-135.

7. Jin, X., Wen, Z., Wang, K., and Xiao, X., "Effect of passenger car curving on rail corrugation at a curved track” Volume 260, Issue 6, 10 March 2006, p619-633.

8. Zhai, W., and Cai, Z., "Dynamic interaction between a lumped mass vehicle and a discretely supported continuous rail track" Computers and Structures Vol. 63, No. 5, pp. 987-997, 1997.
9. Nielsen, J.C.O., and Oscarsson, J., "Simulation of dynamic train-track interaction with state-dependent track properties” Journal of Sound and Vibration, vol. 275 (2004) 515-532.

10. Sun, Y. Q., and Dhanasekar, M., "Importance of the track modeling on the determination of the critical speed of wagons" Vehicle system Dynamics Supplement 41 (2004), p232-241.

11. Rajib Ul Alam Uzzal, "Role of Railway VehicleTrack System and Design Parameters on Flat-Induced Impact Load” M. A. Sc thesis, Dept. of Mechanical \& Industrial Engineering, Concordia University, Montreal, Quebec, Canada 2007.

12. Cai, Z., "Modeling of Rail track dynamics and wheel rail interaction" $\mathrm{Ph} \mathrm{D}$ thesis, Dept. of Civil Engineering, Queen's University, Kingston, Ontario, Canada 1992.

13. Lonsdale, C., Dedmon, S., and Pilch, J., "Effects of increased gross rail load on 36-inch diameter freight car wheels"available at www.standardsteel.com/rdpapers/2001

14. Transport Canada "Railway Freight Car Inspection and Safety Rules" available at http://www.tc.gc.ca/railway/Rules/TC_0-06-1.htm

15. Reilly, K., "Railway Wheelsets", Rail Safety and Standards Board, London, UK, June 2003.

16. Dong, R.G., "Vertical dynamics of railway vehicletrack system," Ph.D. Thesis, Dept. of Mechanical and Industrial Engineering, Concordia University, Montreal, Canada, 1994. 